\title{
Tablet plus Vaginal Tablet Dosage Form
}

National Cancer Institute

\section{Source}

National Cancer Institute. Tablet plus Vaginal Tablet Dosage Form. NCI Thesaurus. Code C149976.

Combination package consisting of a tablet and a vaginal tablet. 